\title{
STRESSED POLYSTYRENE CAUSES INCREASED MEMBRANE SENSITIVITY OF ADHERENT CELLS TO FLUID SHEAR FORCE: TECHNICAL NOTE
}

\author{
J.Y. Lau, R. Tchao* \\ Division of Pharmacology and Toxicology, Department of Pharmaceutical Sciences, University of the Sciences in \\ Philadelphia, Philadelphia, PA, USA
}

\begin{abstract}
Adherent cells transduce signals from the extracellular matrix, which result in changes to various cell functions, including cell spreading and morphology. However, changes to mechanical properties of cell membranes due to adherence to a substratum have not been studied. Adherent Nara Bladder Tumour (NBT) II cells on polystyrene (PS) discs made from pure, atactic polystyrene react differently between the peripheral $1 \mathrm{~mm}$ zone and centre of the disc. After application of a fluid shear force, cells on the peripheral zone resulted in $91.1 \pm 0.8 \%$ cell death due to instantaneous rupture of apical cell membrane, as

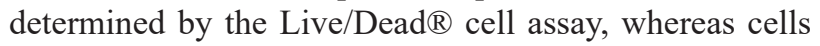
on the disc's centre and surrounding glass surface showed $7.1 \pm 5.7 \%$ and $4.3 \pm 1.7 \%$ cell death, respectively. Under cross-polarized light, the edge of the PS disc showed a low degree of birefringence whereas the centre of the disc did not. We also detached the PS disc and applied various weights $(0.0 \mathrm{~g}$ to $40 \mathrm{~g})$ to the disc at $100^{\circ} \mathrm{C}$ for 2 hours and then cooled rapidly at $4^{\circ} \mathrm{C}$. We found that birefringence developed with stress to PS. NBT II cells grown on stressed PS showed an average of $55.8 \pm 14.1 \%$ cell death after a fluid shear force while cells on the glass surfaces resulted in only $5.0 \pm 2.7 \%$ cell death. Interestingly, increased birefringence is associated with increased lipophilicity on stressed PS, as determined by Nile Red staining. We propose that NBT II cell interaction with certain molecular characteristics of stressed PS results in altered cell membrane sensitivity to mechanical forces.
\end{abstract}

Key Words: Stressed polystyrene, birefringence, anisotropy, cell adhesion, membrane stability, membrane rupture, fluid shear force, lipophilicity.
The interaction between cells and substratum is bidirectional, and has long been recognized as an essential factor that influences cell behaviour (Coppoloni and Dedhar, 2000). Thus, cells are able to modify the substratum and to sense signals from the external environment leading to biochemical and morphological changes to the cell, both of which have been demonstrated in a variety of studies. Nevertheless, there have been only a few studies on cell membrane resulting from cell interaction with substratum.

In many tissues, adherent cells must withstand constant stress by normal physiological forces, such as fluid shear from blood flow or stretching from urinary bladder expansion. The effect of cell adhesion to substratum on the ability of cell membranes to endure such strong mechanical forces is relatively unexplored. Our previous studies showed that squamous epithelial cell lines, such as Nara Bladder Tumour (NBT) II, are sensitive to a fluid shear force on certain areas of various commercial plastic tissue culture flasks (PTCFs) (Tchao, 1996). Under static conditions, cells grew with similar morphology throughout the surface of the flask forming a monolayer. However, if a fluid shear force was created by decanting media or shaking the flask, cells in certain regions of the flask resulted in immediate membrane rupture and cell death, whereas cells grown on amorphous surfaces, such as glass, did not rupture under similar conditions.

PTCFs are manufactured using atactic polystyrene (PS) consisting of a range of molecular weights (MW) and by mould extrusion. In order to study the effect of PS on cells, we have made discs from single MW PS and applied stress to the discs. We have found that apical membranes of cells adherent on stressed PS rupture after fluid shear, suggesting that cell interaction with a specific molecular arrangement of polymeric substrata affects cell membrane stability.

\section{Materials and Methods}

\section{Cell culture}

*Address for correspondence:

R. Tchao

Division of Pharmacology and Toxicology,

Department of Pharmaceutical Sciences,

University of the Sciences in Philadelphia

600 South $43^{\text {rd }}$ Street, Philadelphia, PA 19104, USA.

E-mail: r.tchao@usip.edu
NBT II cells (ATCC, Manassas, VA, USA), a rat bladder squamous carcinoma cell line, were grown in serum-free and antibiotic-free Dulbecco's modified Eagle's medium (DMEM)/F-12/50:50 mix (Mediatech, Inc., Herndon, VA, USA) supplemented with insulin-transferrin-selenite (Sigma, St. Louis, MO, USA). Cells were cultured in commercial PTCFs and incubated at $37^{\circ} \mathrm{C}$ with $5 \% \mathrm{CO}_{2}$. 


\section{Polystyrene (PS) surface preparation and cell seeding}

A 20\% PS solution in chloroform was made from atactic PS of a single MW (MN:270500; MW:284000; MW/ MN:1.05) (Polymer Source, Inc., Dorval, Quebec, Canada) representing the mean $\mathrm{MW}$ of polymer molecules used in PTCFs. A thin, round disc about $1 \mathrm{~cm}$ in diameter was formed after placing 5-6 drops of the PS solution onto a clean glass slide in a closed chamber containing chloroform where it evaporated slowly overnight at room temperature. The edges of the discs were sealed to the glass slide with silicone glue and sterilized with $90 \%$ ethanol.

To stress the PS discs, slides containing the discs were submerged in water to detach the discs from the glass slides. After drying, each disc was held with a paper clip and suspended from a retort stand at one end. At the opposite end, weights of $0.0 \mathrm{~g}, 0.01 \mathrm{~g}, 0.05 \mathrm{~g}, 0.1 \mathrm{~g}, 0.3 \mathrm{~g}, 1 \mathrm{~g}, 5 \mathrm{~g}, 10 \mathrm{~g}$, $20 \mathrm{~g}$, and $40 \mathrm{~g}$ were attached to provide vertical stress to the discs, placed in a dry oven at $100^{\circ} \mathrm{C}$ for 2 hours, and then cooled immediately at $4^{\circ} \mathrm{C}$ overnight. Heat stressed discs without added weight were placed between two glass slides while in the dry oven to prevent the disc from curling. Stressed PS were glued to a clean glass slide with silicone glue and sterilized with $90 \%$ ethanol.

NBT II cells were seeded $\left(1 \times 10^{5}\right.$ cells $\left./ \mathrm{ml}\right)$ onto the PS and surrounding glass surfaces. Adhered cells were incubated at $37^{\circ} \mathrm{C}$ with $5 \% \mathrm{CO}_{2}$ for 24 hours.

\section{Fluid shear force and cell death quantification}

NBT II cells adhered to the PS and glass surface were subjected to a fluid shear force. The slide was held at a $45^{\circ}$ angle and warm $\left(37^{\circ} \mathrm{C}\right)$, serum-free media $(1 \mathrm{ml})$ was pipetted onto the top of the slide creating fluid shear over the cell surface and cell death was quantified immediately using the Live/Dead ${ }^{\circledR}$ cell assay (Molecular Probes) following manufacturer's procedure. Digital images (520 fields, 200-500 cells/field) of live and dead cells on each PS and glass surface were taken at a 40x magnification collected by a Polaroid DMC 2 digital camera attached to a Zeiss Axioskop fluorescent microscope. Using the ImagePro $^{\mathrm{TM}}$ software, live and dead cells in each field were enumerated. The amount of cell viability and cell death was determined by dividing the total number of live and dead cells from each PS and glass surface by total cell count. Results are expressed as percentages. Each experiment was performed 3 times unless stated otherwise.

\section{Scanning electron microscopy (SEM) studies}

NBT II cells were fixed with a $\%$ glutaraldehyde solution in Hanks' Balanced Salt Solution (HBSS) for 2 hours, dehydrated following standard procedures, and air dried using hexamethyldisilizane (HMDS) at room temperature. After gold coating in a sputter coater (Denton Desk II, NJ, USA), SEM images were taken using a JSM840A SEM (JEOL, USA; Peabody, MA).

\section{Birefringence and lipophilicity studies}

Using a dissecting microscope (Zeiss), PS surfaces were observed under cross-polarized light. PS surfaces were also stained with Nile Red $(0.5 \mu \mathrm{g} / \mathrm{ml})$, a fluorescent lipophilic (a)

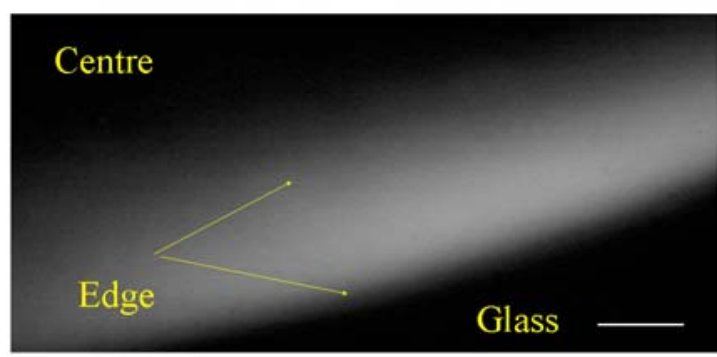

(b)

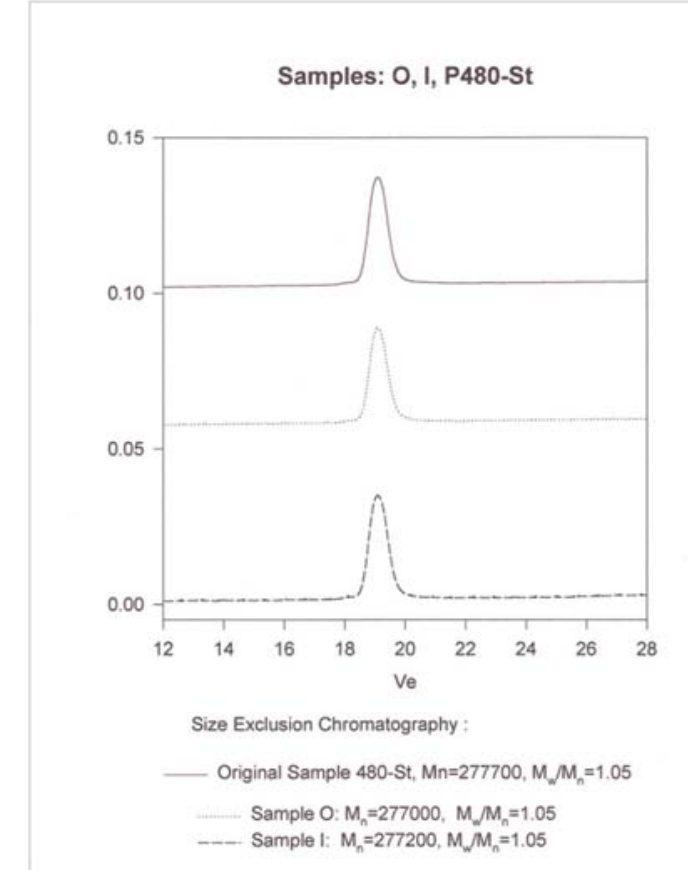

Figure 1: (a) Birefringence image of an unstretched PS disc. Birefringence was seen only on the $1 \mathrm{~mm}$ periphery of an unstressed PS disc, whereas birefringence was not seen on the centre of the disc or the surrounding glass surface $($ Bar $=100 \mu \mathrm{m})$; (b) size exclusion chromatography studies performed on the centre and edge of the PS disc show that there is no difference in the molecular weights of the two areas.

dye. The degree of staining was observed in images taken under a Zeiss Axioskop fluorescent microscope.

\section{Results and Discussion}

In our studies using the PS disc cast from a chloroform solution, a low degree of birefringence was seen on the $1 \mathrm{~mm}$ periphery of the disc, but not on the centre of the disc or glass surface (Fig. 1a). A possibility is that the MWs of PS are different between the edge and the centre. However, size exclusion chromatography of re-dissolved PS from the two areas did not show any differences (Fig. 1b).

NBT II cells seeded onto the PS disc and surrounding glass surface had similar appearance and viability 24 hours after cell seeding. However, after applying a fluid shear force over the surface of adhered cells, a large amount of cell death $(91.1 \pm 0.8 \%)$ occurred on the $1 \mathrm{~mm}$ periphery of the disc, while most of the cells in the disc's centre and 
Table 1 Live/Dead ${ }^{\circledR}$ cell assay results after NBT II cells adhered to a PS and glass surface were subjected to a fluid shear force.

\begin{tabular}{|c|c|c|c|c|}
\hline \multirow{2}{*}{ Surface } & \multicolumn{2}{|c|}{ Polystyrene (PS) } & \multicolumn{2}{c|}{ Glass } \\
\cline { 2 - 5 } & \% Live & \%Dead & \% Live & \%Dead \\
\hline Unstretched PS disc & & & $95.7 \pm 1.7$ & $4.3 \pm 1.7$ \\
\hline Center & $92.9 \pm 5.7$ & $7.1 \pm 5.7$ & & \\
\hline Edge & $8.9 \pm 0.8$ & $91.1 \pm 0.8$ & & \\
\hline 0.01g stretched PS & $32.0 \pm 5.3$ & $68.1 \pm 5.3$ & $96.3 \pm 4.3$ & $3.8 \pm 4.3$ \\
\hline 0.05g stretched PS & $16 \pm 7.6$ & $84.1 \pm 7.6$ & $97.1 \pm 0.9$ & $3.0 \pm 0.9$ \\
\hline 0.1g stretched PS & $46.2 \pm 18.1$ & $53.8 \pm 18.1$ & $99.1 \pm 0.9$ & $1.0 \pm 0.9$ \\
\hline 0.3g stretched PS & $49.7 \pm 17.5$ & $50.3 \pm 17.5$ & $96.5 \pm 0.1$ & $3.6 \pm 0.1$ \\
\hline 1g stretched PS & $37 \pm 11.2$ & $63 \pm 11.2$ & $90.5 \pm 2.8$ & $9.6 \pm 2.8$ \\
\hline 5g stretched PS & $57.1 \pm 0$ & $42.9 \pm 0$ & $92.3 \pm 0$ & $7.7 \pm 0$ \\
\hline 10g stretched PS & $57.5 \pm 0$ & $42.5 \pm 0$ & $95.3 \pm 0$ & $4.7 \pm 0$ \\
\hline 20g stretched PS & $43.8 \pm 5.5$ & $56.2 \pm 5.5$ & $92.9 \pm 4.1$ & $7.1 \pm 4.1$ \\
\hline 40g stretched PS & $59 \pm 0.6$ & $41 \pm 0.6$ & $95.7 \pm 0.8$ & $4.4 \pm 0.8$ \\
\hline
\end{tabular}

Results are expressed as percentages. (An average of 5 to 20 fields containing 200 to 500 cells per field; $n=3$ separate experiments except, $\mathrm{n}=2$ ( $1 \mathrm{~g}$ and $20 \mathrm{~g}$ stretched PS); $\mathrm{n}=1$ (5g and $10 \mathrm{~g}$ stretched PS)).

(a)

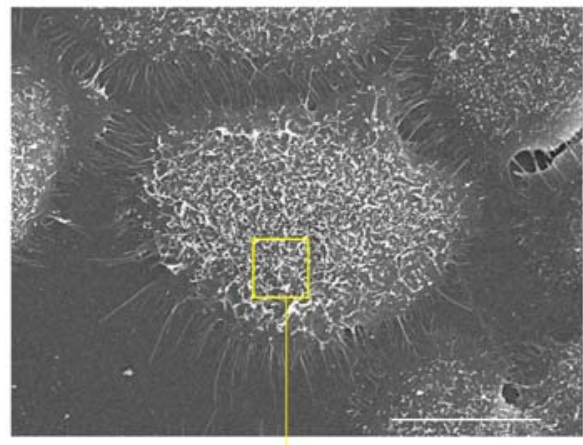

(c)

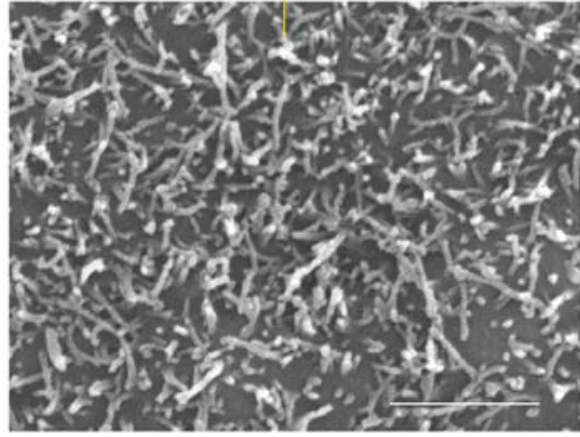

Live Cells (Centre of Disc) (b)

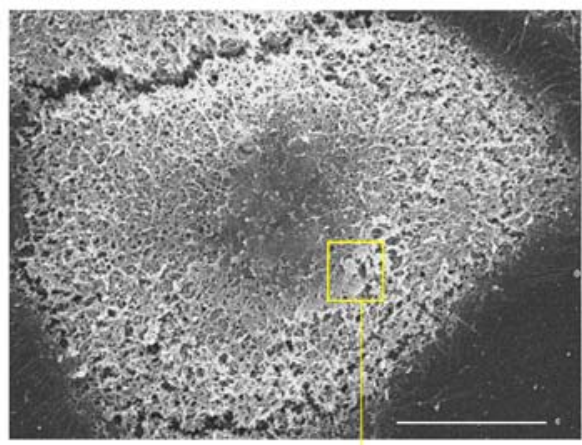

(d)

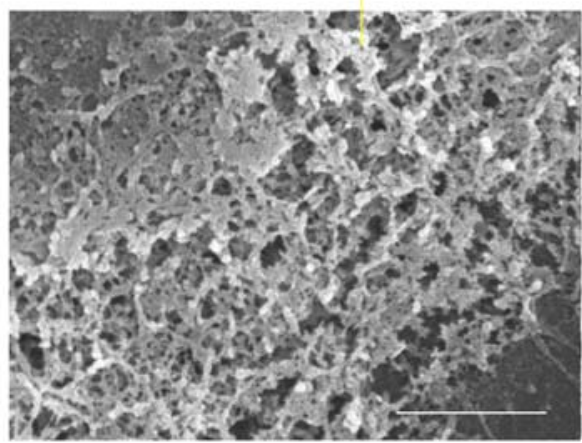

Dead Cells (Edge of Disc)

Figure 2: SEM images of NBT II cells on the $(a, c)$ centre and $(b, d)$ edge of the PS disc after a fluid shear force was applied. At a higher magnification (c,d) cells in the centre of the disc have an intact membrane while cells at the edge have a ruptured membrane. $\mathrm{Bar}=$ (a) $6 \mu \mathrm{m}$, (b) $=4 \mu \mathrm{m}$, (c,d) $1.2 \mu \mathrm{m}$.

surrounding glass surface remained viable $(7.1 \pm 5.7 \%$ and $4.3 \pm 1.7 \%$ cell death, respectively) (Table 1 ). Scanning electron microscopy (SEM) studies showed that cell death was due to apical cell membrane rupture (Fig. 2).

The structure of PS consists of a long hydrocarbon chain with phenyl groups attached to alternating carbon atoms. Studies have indicated that phenyl rings point outward at the free surface of atactic PS (Briggman et al., 2001; Clancy et al., 2001). To induce a unidirectional orientation of phenyl rings, PS surfaces have been rubbed with a velour cloth, which aligns the phenyl rings in the direction of rubbing and produces birefringence under cross-polarized light (Oh-e et al., 2002; Schwab et al.,
2000). The presence of birefringence suggests that there is a certain degree of molecular order or an anisotropic state of the polymer molecules.

Studies have suggested that upon drying, the polymer solution may be subjected to mechanical stress. These forces have been well studied using the "coffee ring" phenomenon by Deegan et al. (1997) who explains that as a drop of coffee is drying, fluid flows outwards from the centre of the drop in order to compensate for evaporation at the edges. As a result, particles in solution are continually transported to the edges where they are deposited producing a "coffee ring". This principle can be applied to the PS disc. As the chloroform is evaporating at the 
edge of the disc, solvent from the centre of the disc is continually flowing towards the edge which may result in a concentration or specific alignment of polymer molecules producing birefringence at the edge of the disc.

Birefringence develops in PS when stressed by temperature (Venerus et al., 1999). We applied different amount of weights $\left(0.0-40 \mathrm{~g}\right.$ weights) to PS discs at $100^{\circ} \mathrm{C}$, creating stress as well as stretch to the disc and, correspondingly, the PS developed a larger area and degree of birefringence. However, the degree of birefringence observed was not uniform over the whole PS specimen. Therefore, we did not pursue further study on birefringence perse.

NBT II cells grown on stressed PS also had similar viable appearance as cells on a glass surface after 24 hours of cell seeding and prior to fluid shear. When a fluid shear force was applied over cells adhered to both surfaces, varying degrees of cell death occurred on PS stressed with $0.01-40 \mathrm{~g}$ weights $(68.1 \pm 5.3 \%, 84.1 \pm 7.6 \%, 53.8 \pm 18.1 \%$, $50.3 \pm 17.5 \%, 63 \pm 11.2 \%, 42.9 \pm 0 \%, 42.5 \pm 0 \%, 56.2 \pm 5.5 \%$, and $41 \pm 0.6 \%$ cell death, respectively), whereas cells on glass surfaces showed only an average of $5.0 \pm 2.7 \%$ cell death (Table 1). Cell death results on heat stressed PS without added weights were similar to unstressed PS discs. Although cells grown on stressed PS resulted in increased membrane sensitivity to fluid shear, the degree of cell death was not directly proportional to the stress applied and was less than that compared to the $1 \mathrm{~mm}$ periphery of the PS disc. This could be due to the non-uniformity of stress applied to the PS disc, which resulted in a varying surface area. As the PS solution dried to form a disc, the density of the PS molecules at the edge may be higher than the centre. Together with stress force, it may produce the optimum alignment of PS molecules to produce the fluid shear force effect on NBT II cell membranes.

Nile Red staining showed that the $1 \mathrm{~mm}$ periphery of the PS disc is lipophilic, which corresponds to the area of birefringence (data not shown). Similarly, stressed PS exhibited increasing, yet non-uniform, degrees of lipophilicity. Polystyrene has generally been regarded as a hydrophobic material. Tretinnikov (2000) suggest that if PS films are cast on hydrophilic substrates, such as glass, a phenyl-rich surface appears at the polymer-air interface. Increased lipophilicity also suggests a change in polymeric molecular arrangement and could cause a different cellsubstratum interaction leading to an apical membrane more sensitive to fluid shear. We are currently studying a lipophilic surface where nearly complete cell death by membrane rupture occurs in response to fluid shear.

The transformation of suspended cells to an adherent morphology during cell adhesion involves major restructuring of the plasma membrane (Pierres et al., 2002). Ertel et al. (1994) has shown that physical cellular interaction with synthetic surfaces, including PS, can result in varying degrees of toxicity. Our study has shown that PS surfaces formed under stress conditions can affect cell membrane sensitivity to fluid shear. It is not known at present whether this effect is due to a change in membrane tensile strength or a change in cytoskeletal organization.

In conclusion, we have shown that NBT II cell membrane interacts differentially between stressed and unstressed polymer molecules, leading to membrane sensitivity to fluid shear. We propose for the first time that NBT II cells may respond to a certain physical orientation (i.e.--anisotropy or lipophilicity) of substrata.

\section{Acknowledgement}

We thank Polymer Source, Inc., Dorval, Quebec, Canada for performing the size exclusion chromatography

\section{References}

Briggman KA, Stephenson JC, Wallace WE, Richter LJ (2001) Absolute molecular orientation distribution of the polystyrene surface. J Phys Chem B 105: 2785-2791.

Clancy TC, Jang JH, Dhinojwala A, Mattice WL (2001) Orientation of phenyl rings and methylene bisectors at the free surface of atactic polystyrene. J Phys Chem B 105: 11493-11497.

Coppolino MG, Dedhar S (2000) Bi-directional signal transduction by integrin receptors. Int J Biochem Cell Biol 32:171-188.

Deegan RD, Bakajin O, Dupont TF, Huber G, Nagel SR, Witten TA (1997) Capillary flow as the cause of ring stains from dried liquid drops. Nature 389: 827-829.

Ertel SI, Ratner BD, Kaul A, Schway MB, Horbett TA (1994) In vitro study of the intrinsic toxicity of synthetic surfaces to cells. J Biomed Mater Res 28: 667-675.

Oh-e M, Hong SC, Shen YR (2002) Orientations of phenyl side-groups and liquid crystal molecules on a rubbed polystyrene surface. Appl Phys Lett 80: 784-786.

Pierres A, Benoliel AM, Bongrand B (2002) Cell fitting to adhesive surfaces: a prerequisite to firm attachments and subsequent events. Eur Cell Mater 3: 31-45.

Schwab AD, Agra DMG, Kim JH, Kumar S, Dhinojwala A (2000) Surface dynamics in rubbed polymer thin films probed with optical birefringence measurements. Macromolecules 33: 4903-4909.

Tchao R (1996) Fluid shear force and turbulenceinduced cell death in plastic tissue culture flasks. In Vitro Toxicology 9: 93-100.

Tretinnikov ON (2000) Hydrophilic (hydrogenbonding) polystyrene surface by substrate-induced surface segregation of benzene groups. Langmuir 16: 2751-2755.

Venerus DC, Zhu S-H, Ottinger HC (1999) Stress and birefringence measurements during the uniaxial elongation of polystyrene melts. J Rheol 43: 795-813.

\section{Discussion with Reviewers}

M. Dalby: The authors mention the flow of solvent in the coffee cup model and say this may lead to stress at the edges of the polystyrene. Could this not, in fact, lead to a build-up of unreacted monomer/slow leaching initiator at the edges? 
Authors: The presence of unreacted monomers can not be ruled out; however, size exclusion chromatography (SEC) studies were performed on the centre and edge portions of the polystyrene (PS) disc (see Fig. 1b). Here, we compared the SEC of the edge portion of the PS disc to the centre. The chromatograms did not reveal additional peaks suggesting the absence of any preferential accumulation of monomers during the drying of the disc. Recently, we asked Polymer Source, Inc. to re-analyze the original PS using SEC and the chromatogram shows one large peak, which represents the single MW of the polystyrene and a smaller peak, which identifies the solvent (Fig. 3). It is still possible that a small amount of unreacted monomer could rise to the surface of the disc. If this were to happen, however, as the PS disc is sterilized with $90 \%$ ethanol before NBT II cells are seeded, the styrene monomers, which are soluble in ethanol, are likely to be washed away.

According to Polymer Source, Inc., the initiator used is secondary butyl lithium and is removed during the purification of the PS.

M. Dalby: Could stressing the polystyrene as described lead to firstly exposure of any unreacted monomer and secondly changes in surface topography? Both of which could effect cells in a wide number of ways.

Authors: We do not believe that any unreacted monomers are present at the surface of the PS disc (See previous comment). However, it is possible that stressing of the PS could lead to changes in surface topography. Although we did not look into the stressed PS, we have attempted to compare the surface topographies of the centre $v s$. the edge of the PS disc by atomic force microscopy (AFM). The results, however, are inconclusive.

Reviewer II: What was the magnitude and direction of the retardance and hence the birefringence? This could have been easily quantified using an appropriate Ehringhaus or similar compensator. Similarly, the fluid shear stress could have been controlled and quantified using a simple laminar flow chamber or rotating disc viscometer method. An interesting experiment that could have been performed would be whether the direction of the flow in relation to the direction of the birefringent slow axis was important. If it were truly the anisotropy (molecular orientation) of the substratum that produced the effect, as concluded by the authors, then such directional effects should be detectable. However, a much simpler and, to my mind, far more likely explanation of the effect is the increasing lipophilicity that the authors observed in the more strained substrata. This implies an increasing hydrophobicity which, in turn, implies a lowered adhesivity for cells irrespective of molecular orientation. Of course lowered adhesiveness and lowered spreading would confer very different properties on the cells in response to shear stress.

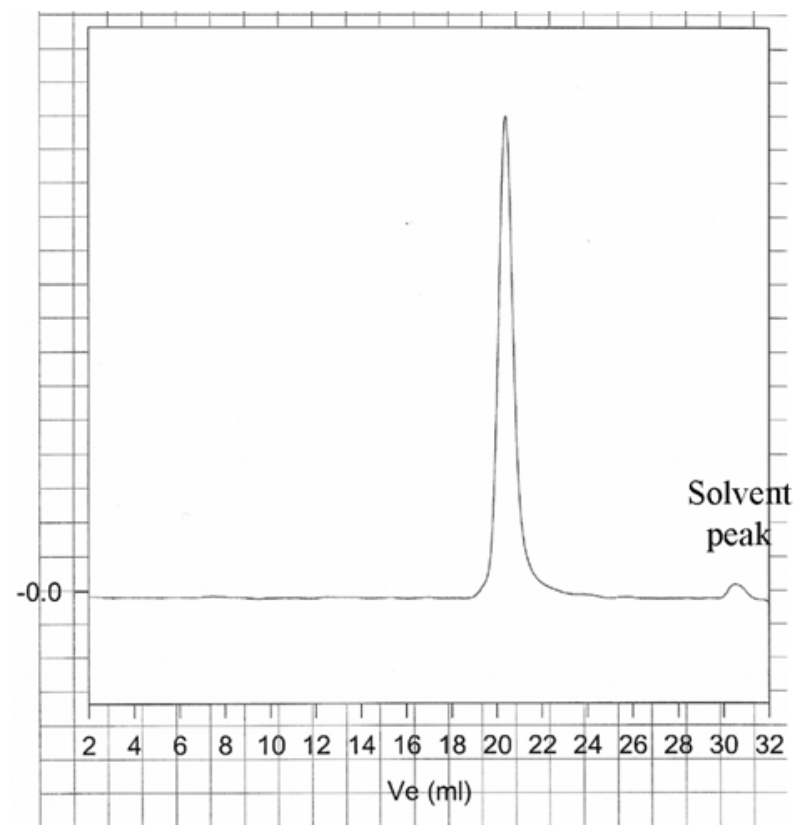

Figure 3: Molecular weight of polystyrene re-analyzed by size exclusion chromatography.

Authors: In this study, the degree of birefringence was not proportional to the degree of cell death. Although we did not measure the birefringence on the various stressed PS, we were able to show that by applying stress to a PS disc, there was an increase in NBT II cell death response to fluid shear. We did not specifically study the relationship between the direction of fluid flow to the direction of the birefringent slow axis. On the PS disc, cell death occurs around the entire perimeter; therefore, if these two parameters correlated, cell death should only occur on certain sections of the edge.

We have also found that the cell death response is possibly dependent on the surface tension of the fluid rather than the force of fluid shear. For example, the addition of $0.01 \%$ Triton-X 100 in the medium completely prevents cell death. Cells shaken vigorously while fully immersed in medium also show no cell death. As suggested by the reviewer, a spinning disc apparatus will certainly determine the precise force involved in rupturing the cell membrane.

From the results in the lipophilicity studies, we are not quite sure if or how birefringence and lipophilcity are related. It could be possible that a certain arrangement of the polystyrene molecules increases the hydrophobicity of the surface. We agree with the reviewer that a hydrophobic surface would change the adhesion and spreading of cells. Since cell death on the PS surface is not uniform; one of two adjacent cells may rupture while the other remains intact, we are investigating a lipid surface that shows more uniformity of cell death in response to fluid shear. This surface would be more predictive and would allow us to study the cellular mechanism underlying membrane rupture in response to fluid shear. 